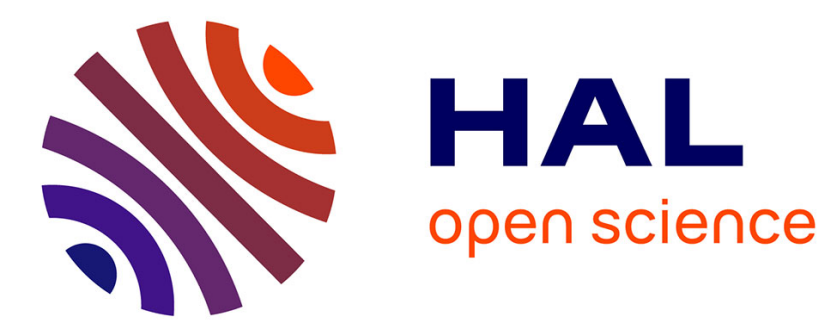

\title{
Sensitivity Evaluation of Subspace-based Damage Detection Method to Different Types of Damage
}

Saeid Allahdadian, Carlos Ventura, Palle Andersen, Laurent Mevel, Michael Döhler

\section{- To cite this version:}

Saeid Allahdadian, Carlos Ventura, Palle Andersen, Laurent Mevel, Michael Döhler. Sensitivity Evaluation of Subspace-based Damage Detection Method to Different Types of Damage. IMAC - 33rd International Modal Analysis Conference, Feb 2015, Orlando, United States. 10.1007/978-3-31915230-1_2 . hal-01109650

\section{HAL Id: hal-01109650 \\ https://inria.hal.science/hal-01109650}

Submitted on 26 Jan 2015

HAL is a multi-disciplinary open access archive for the deposit and dissemination of scientific research documents, whether they are published or not. The documents may come from teaching and research institutions in France or abroad, or from public or private research centers.
L'archive ouverte pluridisciplinaire HAL, est destinée au dépôt et à la diffusion de documents scientifiques de niveau recherche, publiés ou non, émanant des établissements d'enseignement et de recherche français ou étrangers, des laboratoires publics ou privés. 


\title{
Sensitivity Evaluation of Subspace-based Damage Detection Method to Different Types of Damage
}

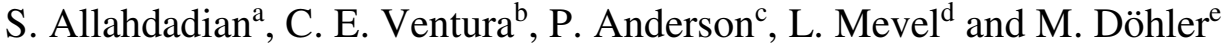 \\ aUniveristy of British Columbia, Vancouver, Canada, saeid@ civil.ubc.ca \\ bUniveristy of British Columbia, Vancouver, Canada, ventura@civil.ubc.ca \\ c Structural Vibration Solutions A/S, Aalborg, Denmark, pa@svibs.com \\ d Inria, Centre Rennes-Bretagne Atlantique, Campus de Beaulieu, 35042 Rennes, France, \\ laurent.mevel@inria.fr \\ e Inria, Centre Rennes-Bretagne Atlantique, Campus de Beaulieu, 35042 Rennes, France, \\ michael.doehler@inria.fr
}

\begin{abstract}
In this paper we investigate a damage detection technique based on the subspace method by applying it to an existing bridge structure model. A reference state of the structure is evaluated using this technique and subsequently its modal parameters are indirectly compared to the current state of the structure. There are no modal parameters estimated in this method. A subspacebased residual between the reference and possibly damaged states is defined independently from the input excitations employing a $\chi^{2}$ test and then is compared to a threshold corresponding to the reference state. This technique is applied to a model of the bridge structure located in Reibersdorf, Austria. The structure is excited randomly with white noise at different locations and the output data is generated at typical locations instrumented and measured in a bridge. Various damages are simulated in different elements and the sensitivity of the method to each type and ratio of damages is assessed. This evaluation is performed by comparing the prediction of the damage state using this technique and the simulated damage of the structure. It can be inferred from the results that in general the statistical subspace-based damage detection technique recognizes most of the damage cases, except the ones with insignificant change in the global dynamic behaviour.
\end{abstract}

Keywords: damage detection, subspace method, health monitoring, bridge damage, statistical damage detection

\section{Introduction}

Structural health monitoring is regarded as the main tool in assessing the functionality of existing structures. The importance of these techniques and researches becomes obvious by considering that failure of a structure can result in catastrophic lost. During past decades extensive researches have been done in the literature in order to investigate an ideal nondestructive damage detection technique. Nondestructive damage detection techniques can be categorized into two groups based on their requirements [1]: (I) local techniques, which need access to all parts of the structure or the location of damage if known, and (II) global damage techniques which use vibration data to evaluate global dynamic characteristics of the structure. In the latter method there is no need to know or have access to the location of damage in priori. The dynamic characteristics used in these methods are usually natural frequencies, mode shapes and damping values.

In order to detect if a damage has occurred in a structure, evaluation of these dynamic characteristics can be avoided by using statistical approaches, e.g. statistical subspace-based damage detection technique (SSDD) [1-5]. The damage can be detected by comparing a statistical model from the possibly damaged structure to the one obtained from a reference state. In other words a subspace based residual function between these states is defined and compared using a $\chi^{2}$ test. In this way there is no need to estimate the natural frequencies and mode shapes of the structure, making this approach capable of being used in real-time 
monitoring of structures. In [3] and [6] it is investigated that this approach can also perform robustly under ambient excitations with changing statistics.

In this paper the performance of the SSDD technique is assessed for different damage types and ratios. A bridge structure, i.e. S101, located at Reibersdorf, Austria, is investigated and simulated for this purpose. This structure was damaged artificially in a progressive manner and it was continuously measured during each damage level [7]. A finite element model of this structure is created and calibrated using the available measured data. This analytical model is damaged in different locations, for different types of elements with different ratios. Subsequently, the structure is excited using white noise excitations. The simulated ambient vibration data is generated by measuring the acceleration time histories of the nodes typically measured in a bridge structure. The simulated data is consequently processed by ARTeMIS software [8] in order to check with the analytical results obtained from the damaged and reference finite element models.

In order to perform the damage detection, the data acquired from each model is processed using the SSDD technique implemented in ARTeMIS softwrae. A reference state is defined by this technique from the data acquired using the undamaged reference model and then the statistics of the data acquired from the damaged structures are compared to a threshold based on the reference state. From this comparison, the sensitivity of the SSDD technique to different types and states of damage is evaluated.

This paper is organized as follows: in section 2 the SSDD technique is described and the formulation of the problem is demonstrated. In section 3, the approach of simulating damage in the analytical model and subsequently simulation of ambient vibration test data is described. These techniques are illustrated in a case study of the realistic model of the bridge in section 4 . The functionality and sensitivity of the SSDD damage detection technique is investigated in that section. In the last section the conclusions are presented.

\section{Stochastic subspace-based damage detection}

The stochastic subspace-based damage detection (SSDD) technique detects the damage in a structure by using a $\chi^{2}$ test on a residual function [4-6]. Therefore in this method, there is no need to compute and compare modal parameters of the reference and possibly damaged states of the system. In other words, this residual function represents the changes occurred to the model which can be caused by a damage in structure.

\subsection{Models and parameters}

The dynamic system of the model can be considered as a discrete time state space model of

$$
\left\{\begin{array}{c}
X_{k+1}=A X_{k}+\varepsilon_{k} \\
Y_{k}=C X_{k}+\vartheta_{k}
\end{array}\right.
$$

where, the state is represented by $X \in \mathbb{R}^{n}$ and the measured output is $Y \in \mathbb{R}^{r} . A$ also represents the state transition matrix and $C$ shows the observation matrix with dimensions $\mathrm{n} \times \mathrm{n}$ and $\mathrm{r} \times \mathrm{n}$, respectively. The state noise, $\varepsilon_{k}$ and measurement noise $\vartheta_{k}$ are assumed to be Gaussian unmeasured white noise with zero mean. The covariance of output measurements $Y_{k}$ can be computed from the state space model (1) by

$$
R_{i}=\mathbf{E}\left(Y_{k+i} Y_{k}^{T}\right)
$$

in which operator $\mathbf{E}$ is the expectation function. With choosing parameters $q$ and $p$ such as $q \geq p+1$, the Hankel matrix $\mathbf{H}$ can be written as

$$
\mathbf{H}=\left(\begin{array}{cccc}
R_{1} & R_{2} & \ldots & R_{q} \\
R_{2} & R_{3} & & R_{q+1} \\
\vdots & & \ddots & \vdots \\
R_{p+1} & R_{p+2} & \ldots & R_{p+q} \\
& & &
\end{array}\right)
$$


As mentioned earlier the measurements are performed in a reference state and a possibly damaged state. The Hankel matrix of the measurements in reference state, $\mathbf{H}_{0}$, can then be computed from (2) and (3). This matrix is then decomposed using singular value decomposition in order to compute the left null space $\mathbf{S}$. Defining $\mathbf{H}$ for the possibly damaged state of the system, the left null space matrix $\mathbf{S}$ in the reference state is characterized by $\mathbf{S}^{T} \mathbf{H}=\mathbf{0}$ ([2-4]). Therefore the residual vector $\zeta_{n}$ can be written as

$$
\zeta_{n}=\sqrt{n} \operatorname{vec}\left(\mathbf{S}^{T} \mathbf{H}\right)
$$

in which, $n$ represents the number of samples measured for computing $\mathbf{H}$. This residual can now be used in order to check if any change is made in the model due to damage. The residual vector $\zeta_{n}$ is asymptotically Gaussian with zero mean in reference state; significant changes in its mean value indicates the structure is moved from its reference state. In order to check this change from the residual vector mean, the $\chi^{2}$ test can be performed as following [4-7].

$$
\chi^{2}=\zeta_{n}^{T} \Sigma^{-1} \zeta_{n}
$$

Herein, $\Sigma$ represents the covariance matrix of the residual in the reference state, and can be shown as

$$
\Sigma=\mathbf{E}\left[\zeta_{n} \zeta_{n}^{T}\right]
$$

It is worth mentioning that the covariance of the input noise $\varepsilon_{k}$ is assumed to not change between the reference state and the possibly damaged state. Details of the covariance computation are found in [3].

By monitoring the value of $\chi^{2}$ and comparing it to a threshold value, the state of the damage of the system can be estimated. This threshold can be simply evaluated using several data sets measured from the structure in its reference state. Subsequently, some other data sets measured from the reference state are used to check the threshold. Then the $\chi^{2}$ value is computed for the possibly damaged structure. If the computed $\chi^{2}$ value is higher than the threshold it can be inferred that the structure may be damaged. In other words, damage that provokes a change in the statistics of the measured data leads to an increase in the $\chi^{2}$ value.

\section{Damage and data simulation}

The ideal test data that can be used to evaluate a damage detection technique is to damage a real structure progressively and measure its response continuously [7]. Having a clear understanding of the conditions of the structure before damaging plays a critical role in the results. Furthermore, in addition to the cost of the procedure, it is not practical to damage a structure and restore it to its undamaged condition for the next test, especially when different elements of the structure are needed to be damaged separately and to various extents.

Simulating the damage in a structure and subsequently generating data that represents the ambient vibration test data can be a useful approach to evaluate damage detection techniques. This data can be an acceptable benchmark to evaluate the functionality of these techniques by allowing control on the test conditions, e.g. structural properties and damage effects. In order to investigate the effect of noise on these techniques, a predefined amount of white noise can be superposed to the data. For simplicity, in this paper there is no additional noise imposed on the results and its effect on the damage detection technique investigated in future study.

In order to evaluate the functionality of the subspace-based damage detection technique, the ambient vibration test data can be simulated for different damage types and amounts. In order to simulate this data, a finite element model of the structure is created and then calibrated to the real structure. It should be mentioned that calibration of the structure does not have a straight effect on the damage detection technique. In other words, the damage detection technique should be able to detect the damage in any structural model including the uncalibrated one as long as the base of comparison is identical. However in this study, calibration to a real structure is performed to obtain a realistic model and simulate the damage in it. The damage in different elements of the model is simulated by reducing the dimensions of one or some short elements in the intended location of damage. The amount of the damage is defined in terms of ratio of this reduction. 
Several points of the structure are excited using white noise excitation in all three directions. Different excitations are imposed on the structure in order to excite the structure as randomly as possible. This excitation can be done by acceleration or load forces in different points of the structure. Subsequently, the simulated data can be obtained by measuring acceleration time histories of the nodes typically measured and instrumented in a bridge.

The simulated data can then be analyzed in order to compute the natural frequencies and their corresponding mode shapes. These can be used to check which mode shapes can be captured by the simulated white noise excitation. Based on the positioning of the sensors and or insufficient excitation of the structure, some mode shapes may not be captured. For the latter, the excitation must be modified to impose to the structure an excitation close to white noise in different points of the structure.

\section{Case study}

Herein, the case study is the model of a bridge structure, namely S101, located in Reibersdorf, Austria. In [7], this structure was progressively damaged and the ambient vibration data was recorded continuously to evaluate statistical damage detection methods. In this study, the finite element model of this structure is used to simulate the damage in more locations of the bridge with various extents. The finite element model is calibrated using the measured data from the bridge to have a precise estimation of the behaviour of structure. The bridge structure and its finite element model are shown in Figure 1. The natural frequencies of the analytical model and the bridge structure are also compared at Table 1.

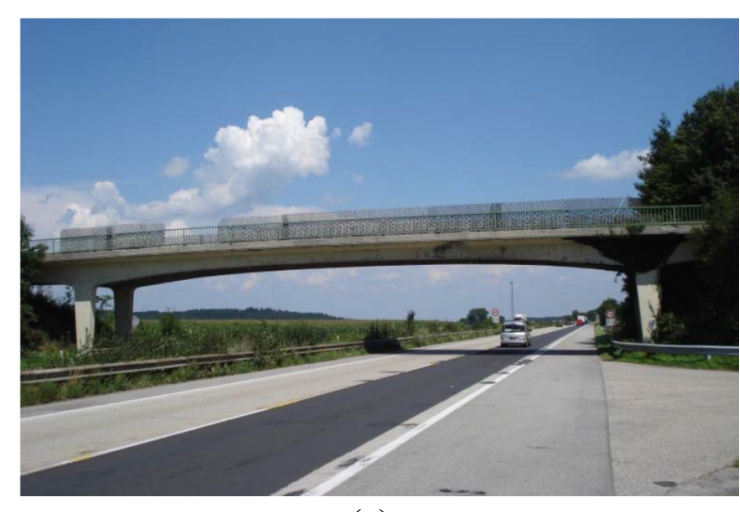

(a)

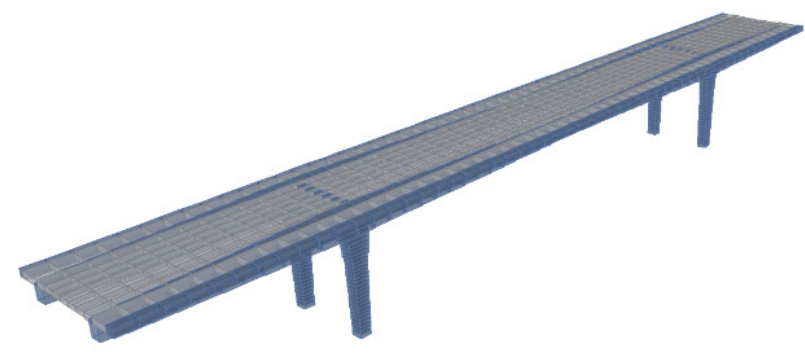

(b)

Fig 1 a S101 bridge structure, Austria, and $\mathbf{b}$ its calibrated finite element model

Table 1 Natural frequencies of the bridge structure in undamaged condition obtained from the measured data and finite element model

\begin{tabular}{lcc}
\cline { 2 - 3 } & $\begin{array}{c}\text { Measured } \\
\text { data }(\mathrm{Hz})\end{array}$ & $\begin{array}{c}\text { Finite element } \\
\text { model }(\mathrm{Hz})\end{array}$ \\
\hline First bending mode & 4.05 & 4.04 \\
First torsional mode & 6.30 & 6.08 \\
Second bending mode & 9.69 & 10.72 \\
Second torsional mode & 13.29 & 12.85 \\
Third bending mode & 15.93 & 19.58 \\
\hline
\end{tabular}

It can be inferred from Table 1 that the finite element model of the structure can be a good representative of the dynamic properties of the bridge. As mentioned in previous section, this calibration is to have a realistic model of a bridge and it does not affect the assessment of the functionality of the damage detection technique.

The effect of bearings in simulating the damage in other elements of the bridge is neglected. However, for simulating the damage in bearings the reference structure is equipped by bearings at the supports. Therefore the reference state of the structure with bearings is used to create the threshold of $\chi^{2}$ and then the damaged models are compared with that reference state. 


\subsection{Damage simulation}

The damage is modeled in different elements of the structure and in different locations. The damaged element types include girders, cap beams, columns, deck and bearings. Furthermore, since this bridge is composed of three spans the damage for the girder and deck is modeled in two locations, i.e. central span and side span. Girders, cap beams and columns are modeled by a number of finite elements. The damage is simulated only in one of these finite elements by reducing a ratio of its section dimension around the strong axis. This damage ratio varies among $20 \%$ (minor damage), $40 \%$ (intermediate damage) and $80 \%$ (severe damage). Damage is simulated in the deck by removing some portions of it and in the bearings by reducing their stiffness.

\subsection{Data simulation}

The finite element model of the structure is excited with a white noise excitation as an acceleration time history in three directions. Moreover, the structure is vibrated by different white noise loads in various locations. The measured points to record acceleration time histories are illustrated in Figure 2a. Spectral densities of the simulated data obtained from undamaged reference case are shown in Figure 2b.

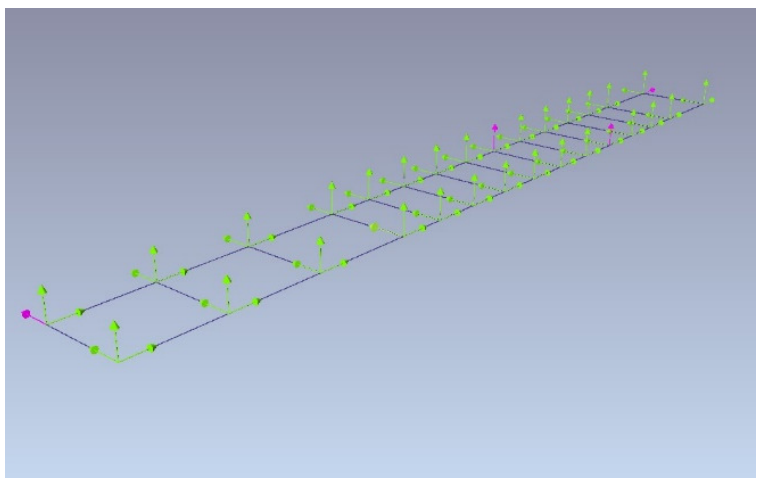

(a)

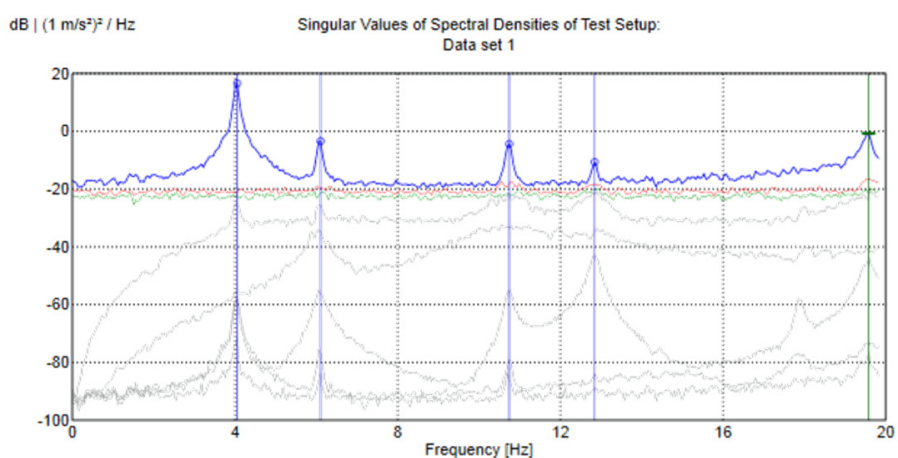

(b)

Fig 2 a measuring-points corresponding to sensor locations; b spectral densities of the singular values of the simulated measurement data in undamaged structure

It can be seen in Figure $2 b$ that the natural frequencies of the analytical model can be obtained from processing the simulated data accurately. Although, the structure is properly excited by white excitation, but some mode shapes cannot be captured. This stems from the location of the sensors and their resolution. As an example, the mode shapes associated to the longitudinal edges of the bridge cannot be captured by the sensors due to their small accelerations occurring in sensor locations.

\subsection{Damage detection}

The undamaged structure is excited for six cases from which four are used to create a threshold for the $\chi^{2}$ value. The two remaining cases are then used to check the threshold. For each damage type and ratio, the simulated data is created and the $\chi^{2}$ test is performed. Subsequently, this value is compared to the computed threshold. The reference state for the structure with fixed supports and with bearings are shown in Figure 3a. In order to validate the reference state, the null space of the Hankel matrix is illustrated in Figure 3b, which shows that only a small portion of the singular values are more than the system order. This suggests that the reference state in both cases are reliable. 


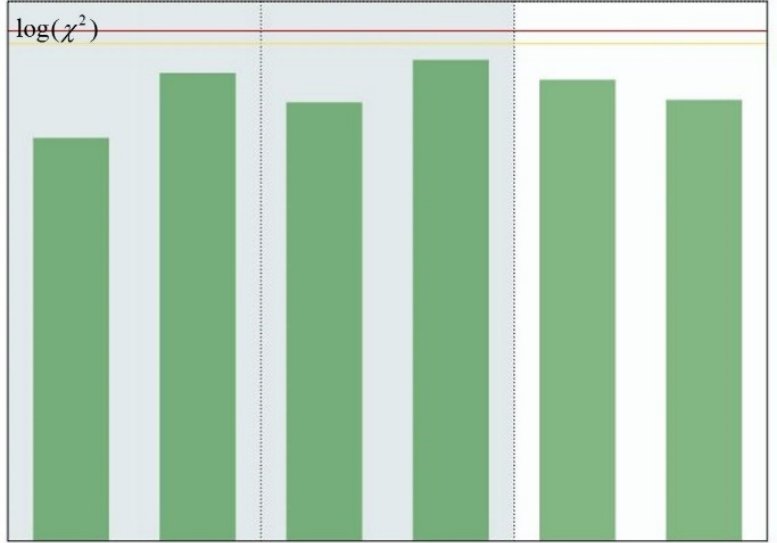

(a)

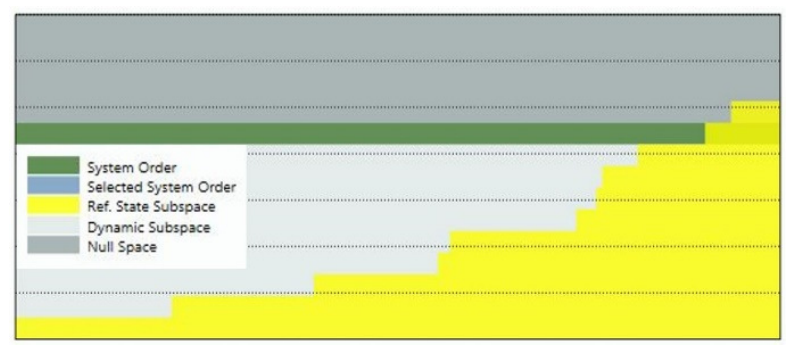

(c)

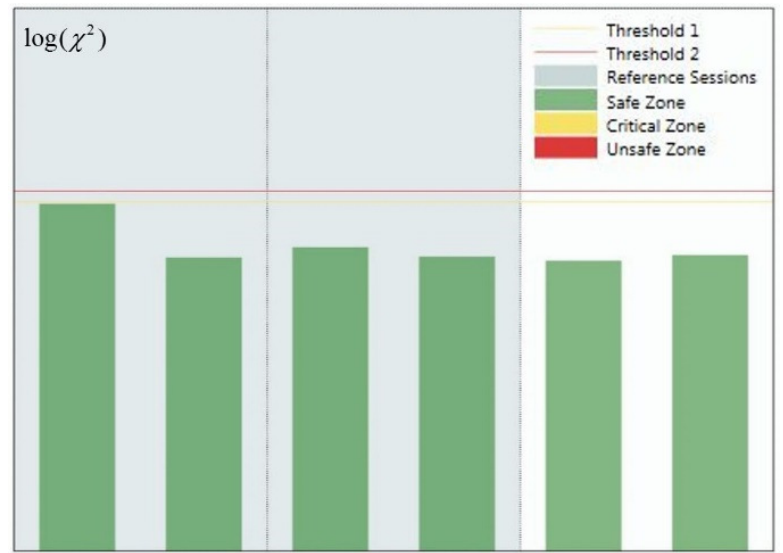

(b)

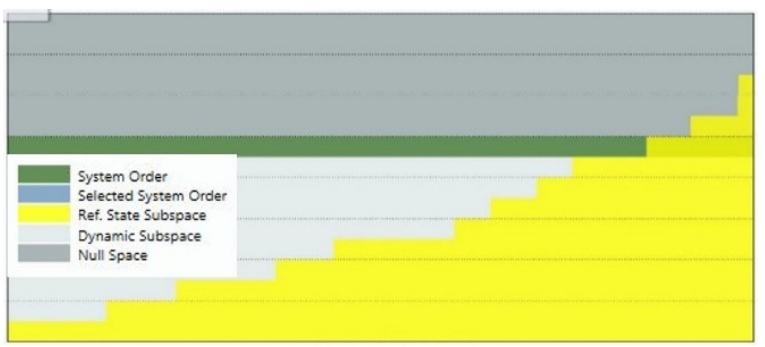

(d)

Fig $3 \chi^{2}$ values and thresholds of the reference state in $\mathbf{a}$ structure with fixed supports and $\mathbf{b}$ with bearings; validation of the reference state in $\mathbf{c}$ structure with fixed supports and $\mathbf{d}$ with bearings

The threshold is computed for two significance values, namely critical zone for significance level $95 \%$ (shown with yellow line in Figure3a) and unsafe zone for significance level 99\% (shown with red line in Figure3b). If the $\chi^{2}$ test value computed from the structure becomes larger than the yellow line, it suggests that the structure is in critical state. Similarly, if this value passes the red line, then the structure is estimated to be in unsafe conditions.

The $\chi^{2}$ test values of the simulated data from different damage cases of the fixed support model are illustrated in Figure 4. For each damage ratio, the $\chi^{2}$ value is computed and compared to the reference state of the structure.

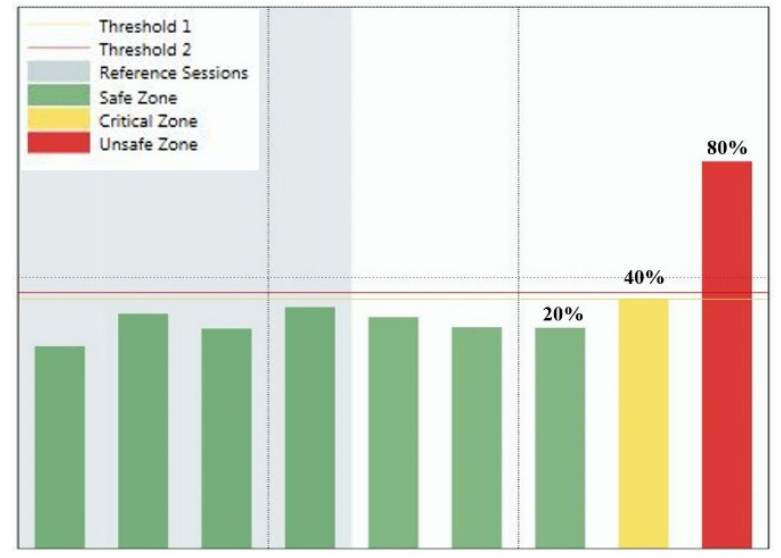

(a)

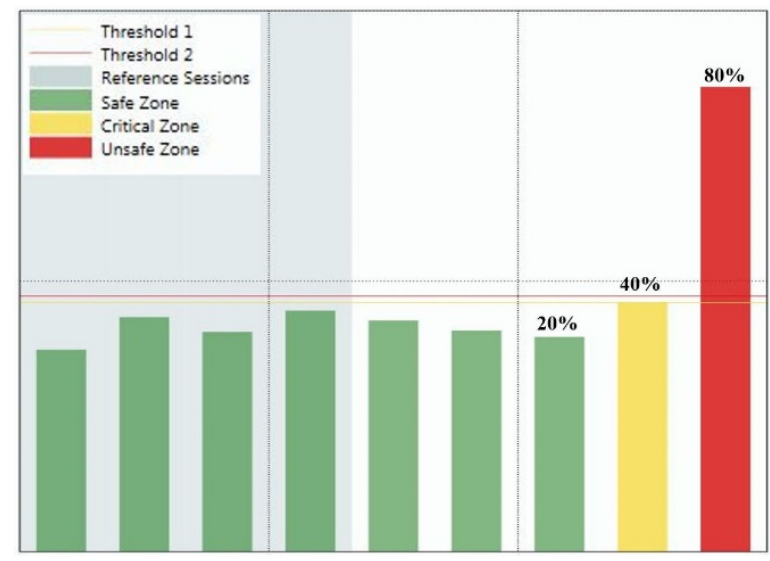

(b) 


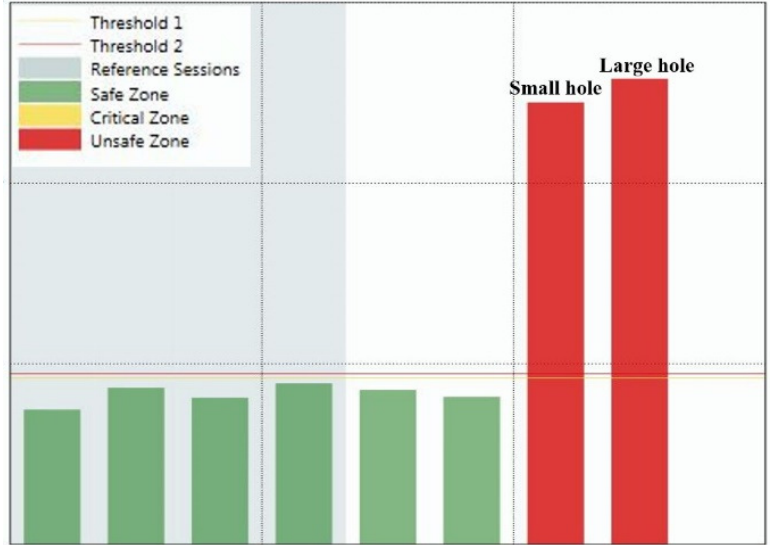

(c)

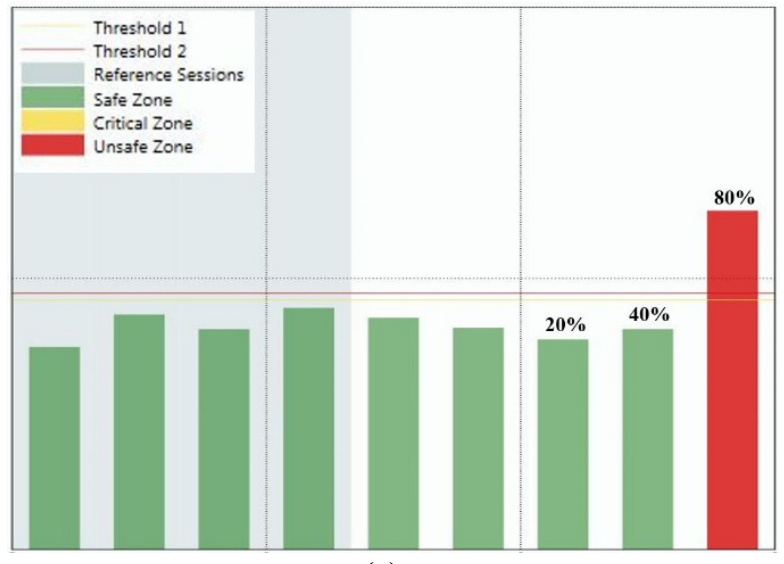

(e)

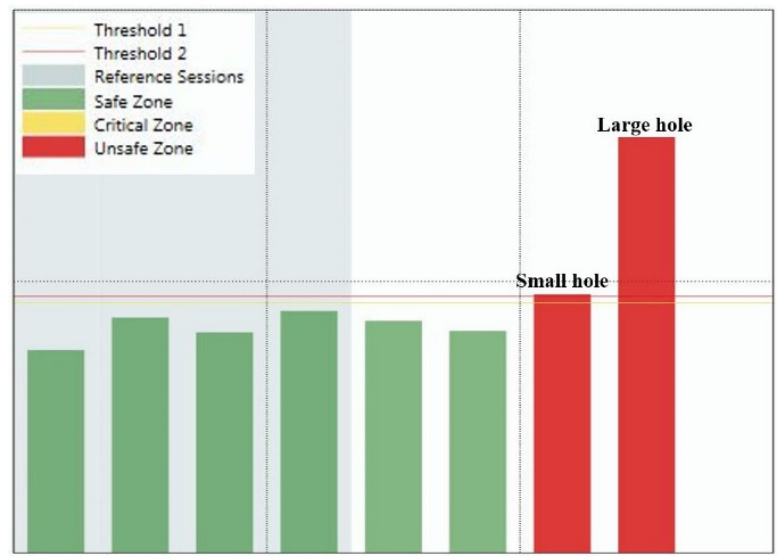

(d)

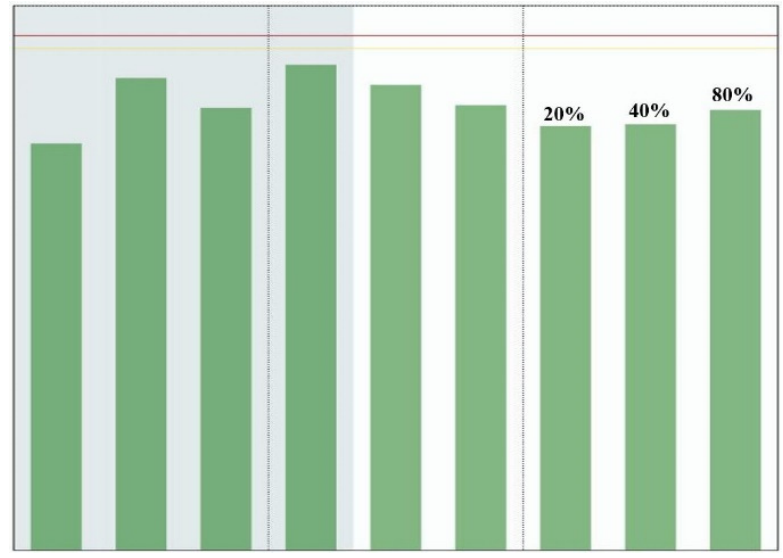

(f)

Fig $4 \chi^{2}$ test from SSDD technique for different element types and damage ratios: a central span girder, b side span girder, $\mathbf{c}$ central span deck, $\mathbf{d}$ side span deck, e column and $\mathbf{f}$ cap beam

It can be seen from Figure $4 \mathrm{a}$ and $4 \mathrm{~b}$, that $\chi^{2}$ value obtained from the girder damage can be captured for the intermediate and severe damage cases only. Minor damage in girder which involves 20\% damage ratio cannot be captured by SSDD technique. The intermediate damage to the girder in both spans is also recognized as critical condition for the structure. Figures $4 \mathrm{c}$ and $4 \mathrm{~d}$ are also showing that the damage to the deck, which was simulated by removal some plane elements of the deck could be captured in all cases. It is worth mentioning by removal of these elements the stiffness of the structure is not changed and the major change happens in mass matrix of the structure, which has a direct effect on dynamic vibration of the structure and can be captured effectively with this technique.

The damage in column can be captured only for the severe case, i.e. damage ratio equal to $80 \%$, as shown in Figure $4 \mathrm{e}$. The cap beam in the structure is located between the columns and connects the girders by crossing them. However, the girders are directly located on top of the columns and therefore cap beams do not have much effect in dynamic behaviour of the model. In all cases the damage in cap beams is not captured suggesting that damage in the elements not affecting the dynamic behaviour of structure may not be recognized by this monitoring technique.

The SSDD technique is used to evaluate damage in bearings as shown in following figure. The resultant $\chi^{2}$ values are compared to the reference state computed for the undamaged structure with bearings. It can be seen that in all cases the damage detection technique can successfully identify the damage in the structure. 


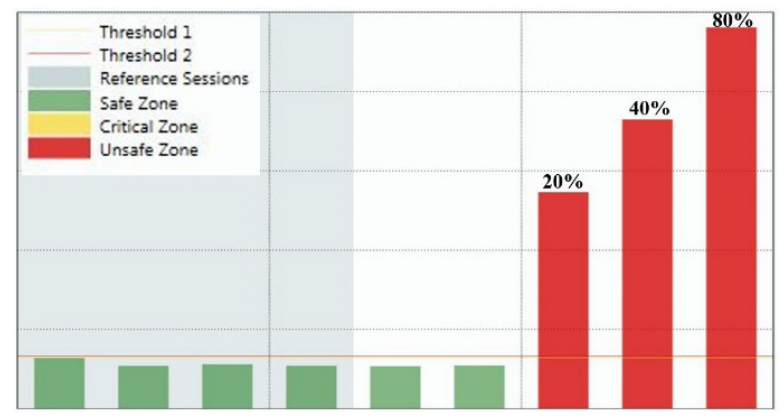

Fig $5 \chi^{2}$ test using SSDD technique for different damage ratios in bearings

It can be seen that in all cases except the cap beam case, the severe damage in the structure could be captured by the SSDD technique. In most of the cases the damage for intermediate damage ratio could be also identified using this technique. For the cap beam element type, as this model was investigated, this element has negligible effect on the dynamic response of the structure. For this reason detecting the damage for that types of elements should be investigated in more details.

\section{Conclusion}

In this paper an analytical finite element model of a bridge structure namely, S101, located at Reibersdorf, Austria, is created and calibrated. The calibration was performed by measured data from the undamaged bridge. This model was employed as a realistic base model to simulate different damage scenarios. The damage was simulated in different element types, including girder, column, bearing, deck and cap beam. In each element type the damage was simulated separately and for different ratios. The damage ratio varied among minor (20\%), intermediate (40\%) and severe (80\%) conditions. For each case, a time history analysis was performed with white noise excitation imposed on the structure.

The ambient vibration test data was simulated by recording the acceleration time histories of the nodes typically measured in this type of structure. This simulated data was used as an input to the statistical subspace-based damage detection technique in order to estimate the damage condition. It was shown that this technique could detect the severe damage condition for all cases except cap beam damage. Moreover, in most of the cases the intermediate ratio damage was recognized successfully. It can be confirmed from the results that this technique cannot detect damages which are not affecting the dynamic behaviour of structure, e.g. damages in cap beams, which is the case for all global vibration-based methods. Future study is intended to investigate the effect of noise on data, more types of damage cases and ratios.

\section{References}

[1] Fan, Wei, and Pizhong Qiao. "Vibration-based damage identification methods: a review and comparative study." Structural Health Monitoring 10.1, 83-111, (2011).

[2] Basseville, Michele, Maher Abdelghani, and Albert Benveniste. "Subspace-based fault detection algorithms for vibration monitoring." Automatica 36.1, 101-109, (2000).

[3] Döhler, Michael, Laurent Mevel, and Falk Hille. "Subspace-based damage detection under changes in the ambient excitation statistics." Mechanical Systems and Signal Processing 45.1, 207-224, (2014).

[4] Döhler, Michael, and Laurent Mevel, "Subspace-based fault detection robust to changes in the noise covariances". Automatica, 49.9,2734-2743, (2013).

[5] Döhler, Michael. Subspace-based system identification and fault detection: Algorithms for large systems and application to structural vibration analysis. Diss. Université Rennes 1, (2011).

[6] Döhler, Michael, and Falk Hille. "Subspace-based damage detection on steel frame structure under changing excitation." Structural Health Monitoring, Volume 5. Springer International Publishing. 167-174, (2014).

[7] Döhler, Michael, et al. "Structural health monitoring with statistical methods during progressive damage test of S101 Bridge." Engineering Structures 69, 183-193, (2014).

[8] Structural Vibration Solutions, http://www.svibs.com/. 